\title{
Implicación del circuito corticoestriadotalámico en pacientes con trastorno obsesivo-compulsivo durante una tarea de control inhibitorio con contingencias de recompensa y castigo
}

Josep Pena-Garijo, Alfonso Barros-Loscertales, Noelia Ventura-Campos, M. Ángeles Ruipérez-Rodríguez, Silvia Edo-Villamón, César Ávila

Introducción. Estudios recientes sobre neuroimagen en el trastorno obsesivo-compulsivo (TOC) muestran alteraciones en el circuito frontoestriadotalámico, lo que daría lugar a una disfunción ejecutiva. Éste podría ser el sustrato neurocognitivo de los síntomas principales del TOC: obsesiones y compulsiones.

Sujetos y métodos. Se comparó tanto la activación cerebral como el rendimiento conductual de un grupo de 13 pacientes con TOC comparado con un grupo control de 13 sujetos sanos a través de resonancia magnética funcional durante la ejecución de una tarea de control inhibitorio con contingencias de recompensa y castigo. Los efectos de la medicación también se analizaron.

Resultados. Los análisis intragrupo mostraron un tiempo de reacción más largo durante la condición de go/no go en ambos grupos, aunque no hubo diferencias entre los grupos en la ejecución de la tarea. En relación con dicha tarea, se observó, en los sujetos sanos, una activación significativa de grandes áreas del cerebelo y de los lóbulos occipital, temporal y parietal. En comparación con los controles, los pacientes obsesivos mostraron una activación reducida en los giros frontal medial y superior derechos, la corteza cingulada anterior y el núcleo caudado, y una mayor activación en la circunvolución parietal inferior y el giro fusiforme. Se encontraron efectos de la medicación en la corteza frontal y estructuras basales.

Conclusiones. Estos resultados están de acuerdo con el argumento de que la disfunción en el sistema corticoestriado en el TOC se asocia a una disminución de la actividad cerebral en respuesta a tareas cognitivas.

Palabras clave. Control inhibitorio. Neuroimagen funcional. Tarea cognitiva. Trastorno obsesivo-compulsivo.

\section{Introducción}

El trastorno obsesivo-compulsivo (TOC) es una condición clínica heterogénea. La conceptualización de las obsesiones y compulsiones ligadas al trastorno ha ido variando, desde las tradicionales propuestas categoriales planteadas por la American Psychiatric Association en el Manual diagnóstico y estadístico de los trastornos mentales, cuarta edición, texto revisado (DSM-IV-TR) [1], a las propuestas dimensionales planteadas por Mataix-Cols et al [2], entre otros. Breiter y Rauch [3] fueron los primeros en proponer la implicación de los circuitos corticoestriado, talámico y límbico en el TOC, mediante la utilización de resonancia magnética funcional (RMf). La investigación hasta la fecha parece reafirmarse en la línea de la disfunción frontoestriatal, tanto con técnicas estructurales [4], análisis morfométri- $\cos$ [5-12], como con técnicas funcionales [13]. Mataix-Cols et al [2] sugieren que estos hallazgos, en ocasiones contradictorios, podrían reconceptualizarse como déficits cognitivos secundarios a disfunciones del sistema ejecutivo, e insta a investigar en el futuro con paradigmas que combinen neuropsicología e imagen funcional.

En un intento de aclarar los aspectos de conectividad del circuito frontocingulado, Schlösser et al [14] demostraron que se produce una hiperactividad en el sistema de control de errores en sujetos con TOC relacionado con la supresión de respuestas prepotentes durante una tarea de toma de decisiones. Observan, además, la relación entre la tarea y una mayor conectividad entre el cíngulo anterior y la corteza prefrontal dorsolateral en los pacientes obsesivos. Asimismo, el grupo de Nakao [15] analizó diferentes componentes cognitivos mediante prue-
Departamento de Psicología Básica, Clínica y Psicobiología. Universitat Jaume I. Castelló de la Plana, España.

Correspondencia: Dr. Josep Pena Garijo. Departamento de Psicología Básica, Clínica y Psicobiología. Facultad de Ciencias Humanas y Sociales. Universitat Jaume I. Campus Riu Sec. Avda. Sos Baynat, s/n. E-12071 Castelló de la Plana.

E-mail:

jpena@psb.uji.es

Aceptado tras revisión externa: 22.03.11.

Cómo citar este artículo: Pena-Garijo J, Barros-Loscertales A, Ventura-Campos N, RuipérezRodríguez MA, Edo-Villamón S, Ávila C. Implicación del circuito corticoestriadotalámico en pacientes con trastorno obsesivo-compulsivo durante una tarea de control inhibitorio con contingencias de recompensa y castigo. Rev Neurol 2011; 53: 77-86.

(c) 2011 Revista de Neurología 
Tabla I. Datos demográficos y evaluación de la muestra (media \pm desviación estándar).

\begin{tabular}{lcc}
\hline & Controles $(n=13)$ & Pacientes $(n=13)$ \\
\hline Sexo (M/F) & $5 / 8$ & $6 / 7$ \\
\hline Edad (años) & $37,14 \pm 9,79$ & $37,08 \pm 11,08$ \\
\hline Nivel de estudios a & $2 \pm 1$ & $1,86 \pm 1,07$ \\
\hline Y-BOCS & $12,71 \pm 2,75$ & $25,38 \pm 5,45$ \\
\hline $\begin{array}{l}\text { Vocabulario } \\
\text { Cubos }\end{array}$ & $9,57 \pm 3,73$ & $11,61 \pm 1,66$ \\
\hline BDI-II & $7,43 \pm 3,69$ & $10,61 \pm 3,88$ \\
\hline $\begin{array}{l}\text { BDI-II: inventario para la depresión de Beck-II; Y-BOCS: escala Yale-Brown para trastorno obsesivo-compulsivo. } \\
\text { a 1. Primarios. 2. Secundarios. 3. Superiores. }\end{array}$ & $16,38 \pm 11,03$ \\
\hline
\end{tabular}

bas neuropsicológicas y el uso de la RMf en pacientes afectos de TOC. Los pacientes obtuvieron puntuaciones más bajas en recuerdo diferido y en recuerdo inmediato al ser comparados con los controles. En la RMf, los pacientes mostraron una mayor activación en el córtex prefrontal dorsolateral derecho, giro temporal superior izquierdo, ínsula izquierda y cuneus. La conclusión a la que llegan es que existen anormalidades tanto neuropsicológicas como cerebrales en los pacientes con TOC. En esta misma línea, el grupo de Chamberlain [16] propone que existe un control inhibitorio alterado en los pacientes con TOC. Las anormalidades en el circuito corticosubcortical, particularmente en el circuito orbitofrontal lateral, podrían representar las bases neurobiológicas de estos fallos inhibitorios. Por último, Roth et al [17] encontraron también hipoactivación en áreas del circuito frontoestriadotalámico en los pacientes con TOC al ejecutar tareas de inhibición de respuestas.

En el presente trabajo, analizaremos las diferencias en la activación cerebral entre un grupo de pacientes diagnosticados de TOC y un grupo de sujetos sanos al realizar una tarea de control inhibitorio con recompensa variable (go/no go rewarded task), siguiendo un paradigma similar al propuesto por Ávila [18] y adaptado a la RMf. Esperamos encontrar que esta tarea implicará el circuito corticoestriadotalámico por la conjunción de los procesos de inhibición de respuesta y de procesamiento de la recompensa, así como una respuesta diferencial de este circuito en pacientes con diagnóstico de TOC en relación con el grupo control.

\section{Sujetos y métodos}

\section{Descripción de la muestra}

Los participantes fueron 13 pacientes adultos con TOC y 13 adultos sanos equiparados en edad, sexo y nivel educativo. Los pacientes se reclutaron en la Unidad de Salud Mental del Hospital General de Castelló. Todos ellos fueron informados verbalmente y por escrito, y firmaron un consentimiento informado previo al inicio de la investigación.

Todos los participantes completaron las subescalas de cubos y vocabulario de la escala de inteligencia de Wechsler para adultos (WAIS III) [19], y aquéllos que puntuaron por debajo de la puntuación típica de 6 en cualquiera de los substests fueron excluidos del estudio. Los pacientes se diagnosticaron de acuerdo con los criterios del DSM-IV-TR [1], empleando la Structured Clinical Interview for DSM-IV Axis I Personality Disorders [20]. Los sujetos controles sanos fueron excluidos si presentaban historia de enfermedad mental. Seis pacientes presentaron exclusivamente criterios de TOC, pero, entre los otros siete, se encontraron diagnósticos de fobia social (dos), depresión mayor o distimia (tres), fobia específica (dos) y trastorno de personalidad (cinco). Ocho pacientes estaban tomando medicación antidepresiva (inhibidores de la recaptación de serotonina) y cinco estaban libres de medicación al menos cuatro semanas antes de la prueba de neuroimagen. La escala Yale-Brown para TOC (Y-BOCS) [21] se usó para evaluar la gravedad de los síntomas en el grupo de pacientes. Todos los sujetos respondieron el inventario para la depresión de Beck-II (BDI-II) [22] para evaluar el estado de ánimo. Los datos de la evaluación se resumen en la tabla I. La historia de enfermedad neurológica, traumatismo craneal con pérdida de conciencia, trastorno por abuso de sustancias, condiciones médicas que pudieran influir en el funcionamiento cognitivo o factores que contraindicaran la aplicación de la RM se utilizaron como criterios de exclusión del estudio.

\section{Tarea}

La tarea consistió en 132 ensayos y se aplicó un diseño de bloques. Los participantes tenían que responder diferencialmente en dos teclas en función de la dirección señalada por una flecha que aparecía en el centro de la pantalla. Simultáneamente, en la parte superior de la pantalla aparecían unos estímulos (círculos) que cambiaban de color. Cada respuesta se recompensó en función del tiempo de reacción, es decir, cuanto más rápida era la respuesta, 
mayor puntuación obtenía, y, por tanto, mayor recompensa. La fórmula para calcular la ganancia (G) como una función del tiempo de reacción (TR) era $\mathrm{G}=1.100 / \mathrm{TR}$.

Los participantes completaron 12 bloques de 30 segundos, y cada bloque estaba formado por 11 ensayos. La duración de cada ensayo era de 2,72 segundos. En la condición de control, los sujetos debían responder con la máxima rapidez. Si se equivocaban en la dirección de la flecha, perdían 10 puntos. Por su parte, en la condición de activación, los círculos de colores preparaban a la persona para la respuesta de inhibición, ya que los participantes debían inhibir su respuesta cuando se presentaba un círculo rojo (el 18\% de los ensayos, dos veces por bloque, un total de 12 círculos rojos). Si los participantes no inhibían su respuesta ante un círculo rojo, recibían un castigo, que consistía en la pérdida de la mitad de los puntos acumulados (Fig. 1).

\section{Adquisición de las imágenes}

Las imágenes se obtuvieron en un escáner Siemens Avanto (Erlangen, Germany) de 1,5 T. Los participantes se acomodaron en decúbito supino dentro del escáner de RM. Los estímulos fueron presentados directamente mediante unas gafas Visuastim XGA con una resolución de $800 \times 600$ (Resonance Technologies). Para cada participante, se adquirió un volumen estructural axial de 176 cortes que ofreciera información anatómica de alta resolución (FSPGR; tiempo de eco, TE = 4,9 ms, tiempo de repetición, TR $=11 \mathrm{~ms}$, campo de visión $=24 \mathrm{~cm}$; matriz $=256 \times 224 \times 176$, tamaño del vóxel $=1 \times$ $1,04 \times 1,04)$. Posteriormente, se adquirió una secuencia de gradiente ecoplanar $\mathrm{T}_{2}$ (EPI) funcional (matriz $=64 \times 64, \mathrm{TE}=50 \mathrm{~ms}, \mathrm{TR}=3.000$, grosor de corte $=5 \mathrm{~mm}$, espacio entre cortes $=0 \mathrm{~mm}$, ángulo $=90^{\circ}$, tamaño del vóxel $=3,94 \times 3,94$ ). Se adquirieron 29 cortes axiales paralelos a la comisura anterior-posterior.

\section{Análisis estadísticos}

Los datos conductuales se analizaron con el programa SPSS v. 15.0 mediante pruebas adecuadas a sus características de distribución. Las imágenes de resonancia fueron procesadas utilizando el programa SPM5 (Wellcome Department of Cognitive Neurology, Londres). Todos los volúmenes fueron realineados en función del primero. Se realizó una corrección de los artefactos de movimiento y un corregistro sobre su correspondiente anatómico, aplicando transformación de 12 parámetros afines. Des-
Figura 1. Vista en pantalla de la tarea de control inhibitorio.

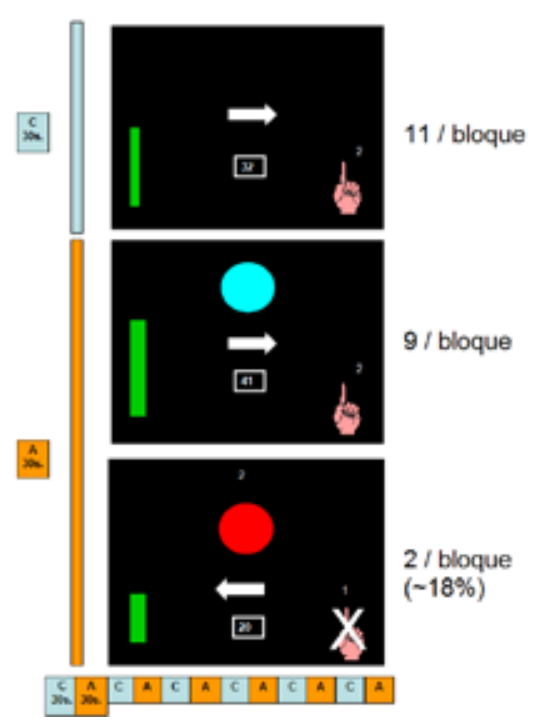

pués, las imágenes fueron normalizadas sobre un espacio estereotáxico estandarizado -coordenadas del Montreal Neurology Institute (MNI)-, y sobre los datos funcionales se aplicó un suavizado espacial de $8 \mathrm{~mm}$ con una función gaussiana. Los datos para cada participante se modelaron mediante un diseño de bloques con la función de la respuesta hemodinámica.

El análisis estadístico fue realizado individual y grupalmente utilizando el modelo lineal general [23]. El análisis de grupo fue realizado utilizando el modelo de efectos aleatorios que nos permite hacer inferencia sobre la población general. La activación del grupo para la condición de control inhibitorio se obtuvo con una prueba $t$ para una muestra $(p<$ 0,001 , no corregida) y con una extensión mínima de 20 vóxeles. Esta combinación de umbral estadístico y extensión de cluster para determinar la significación se seleccionó de acuerdo con estudios previos [24], dado lo modesto del tamaño de la muestra. Las hipótesis sobre diferencias entre grupos fueron evaluadas aplicando un ANOVA de un factor $(p<$ 0,005 , sin corregir por múltiples comparaciones) para activaciones de una extensión mínima de 20 vóxeles continuos. No se aplicó corrección de Bonferroni para múltiples comparaciones, dado el escaso tamaño y la heterogeneidad de la muestra $[17,25$, 26]. Las coordenadas para cada pico de máxima significación en la activación fueron convertidas des- 
Tabla II. Datos de la ejecución en las dos condiciones de la tarea de control inhibitorio.

\begin{tabular}{lccccc}
\hline & $\begin{array}{c}\text { A. Condición de control } \\
\text { (sólo recompensa) }\end{array}$ & $\begin{array}{c}\text { B. Condición de } \\
\text { control inhibitorio }\end{array}$ & $\begin{array}{c}\text { \% de errores en } \\
\text { eluicio de dirección } \\
\text { (condición A) }\end{array}$ & $\begin{array}{c}\text { \% de errores en } \\
\text { el juicio de dirección } \\
\text { (condición B) }\end{array}$ & $\begin{array}{c}\text { \% de comisiones } \\
\text { (condición B) }\end{array}$ \\
\hline Controles & $388 \pm 65 \mathrm{~ms}$ & $445 \pm 89 \mathrm{~ms}$ & $0,58 \pm 0,98$ & $0,42 \pm 0,81$ & $3,84 \pm 5,5$ \\
\hline Pacientes & $398 \pm 68 \mathrm{~ms}$ & $440 \pm 64 \mathrm{~ms}$ & $0,11 \pm 0,42$ & $0,28 \pm 0,69$ & $5,76 \pm 7,89$ \\
\hline
\end{tabular}

de el sistema MNI aplicado por SPM5 a coordenadas de Talairach para, posteriormente, ser localizadas usando el atlas neuroanatómico de Talairach y Tournoux [27].

\section{Resultados}

Los grupos no difirieron en edad, sexo o nivel educativo $(p>0,1)$. Las subescalas de vocabulario y cubos del WAIS-III tampoco difirieron entre grupos. El grupo de pacientes puntuó más alto que los controles en el BDI-II $(t=2,065 ; p=0,042)$. La puntuación media total del Y-BOCS para el grupo con TOC estuvo en el rango de gravedad moderada. El resumen de estos datos se puede ver en la tabla I.

La ejecución de la tarea mostró diferencias en el tiempo de reacción relacionado con el juicio de dirección en ambas condiciones, tanto dentro de cada grupo (TOC: $t=4,71 ; p=0,001$; controles: $t=4,44$; $p=0,001)$, como entre ellos $(t=6,35 ; p<0,001)$, siendo más lentos en la condición de control inhibitorio ( $g o /$ no go) que en la de sólo recompensa ( $g o$ ). Sin embargo, estas diferencias no fueron significativas en la precisión del juicio de dirección (derechaizquierda) ( $t=0,82 ; p=0,27)$, cometiendo muy pocos errores. Por otro lado, los grupos no difirieron en tiempo de reacción al compararlos en cada una de las condiciones $(t=0,39 ; p=0,86)$ o en la diferencia en el tiempo de reacción entre condiciones $(t=0,95 ; p=0,32)$. Ambos grupos fueron también equivalentes en el porcentaje de errores de comisión durante la tarea de control inhibitorio $(t=0,72$; $p=0,31)$. La tabla II muestra un resumen de estos datos conductuales.

\section{Análisis intragrupo}

Las tablas III y IV y la figura 2 muestran las regiones donde se observan efectos significativos en la activación en cada grupo durante la condición de control inhibitorio (go/no go). Los sujetos sanos mostraron una activación significativa en grandes áreas que se extendían a través del cerebelo y los lóbulos parietal y temporal. Dentro de este grupo se encontraron activaciones más focalizadas en el cuerpo del caudado de forma bilateral, el tálamo, cuerpos mamilares, hipotálamo, cíngulo anterior, y los giros frontales medial y superior. También se encontraron activaciones lateralizadas a la izquierda en el córtex frontal ventrolateral, y el giro precentral (Tabla III). Por otro lado, el grupo de pacientes con TOC también mostró dos grandes clusters de activación que se extendían desde el cerebelo a los lóbulos occipital, temporal y parietal. Se encontraron activaciones más focalizadas tan sólo en el lóbulo frontal derecho, incluyendo los giros medial y superior (Tabla IV).

\section{Comparación entre grupos}

La tabla $\mathrm{V}$ y la figura 2 muestran las regiones donde se observaron diferencias significativas entre ambos grupos. El grupo control tuvo mayores activaciones que el grupo de pacientes con TOC durante la condición de control inhibitorio (go/no go) en el cuerpo del caudado (de forma bilateral), el cíngulo anterior izquierdo y los giros frontales superior y medial. Por otro lado, el grupo de pacientes activó en mayor medida que el control los giros lingual y fusiforme izquierdos, el lóbulo parietal inferior izquierdo, y cuneus y precuneus derechos.

\section{Efectos de la medicación}

Para examinar los efectos de la medicación en nuestros hallazgos, comparamos la activación cerebral durante la tarea de go/no go en los subgrupos de pacientes medicados y no medicados (Tabla V). Las comparaciones revelaron que los no medicados mostraron una mayor activación en el cíngulo anterior izquierdo y el córtex frontal medial adyacente, la ínsula derecha, el giro frontal ventromedial (bilateralmente), el polo temporal izquierdo, el tálamo, la cabeza del caudado izquierdo, el precuneus y el giro 
Tabla III. Regiones que mostraron activación cerebral durante la ejecución de la tarea de control inhibitorio con contingencias de reforzamiento en el grupo de controles.

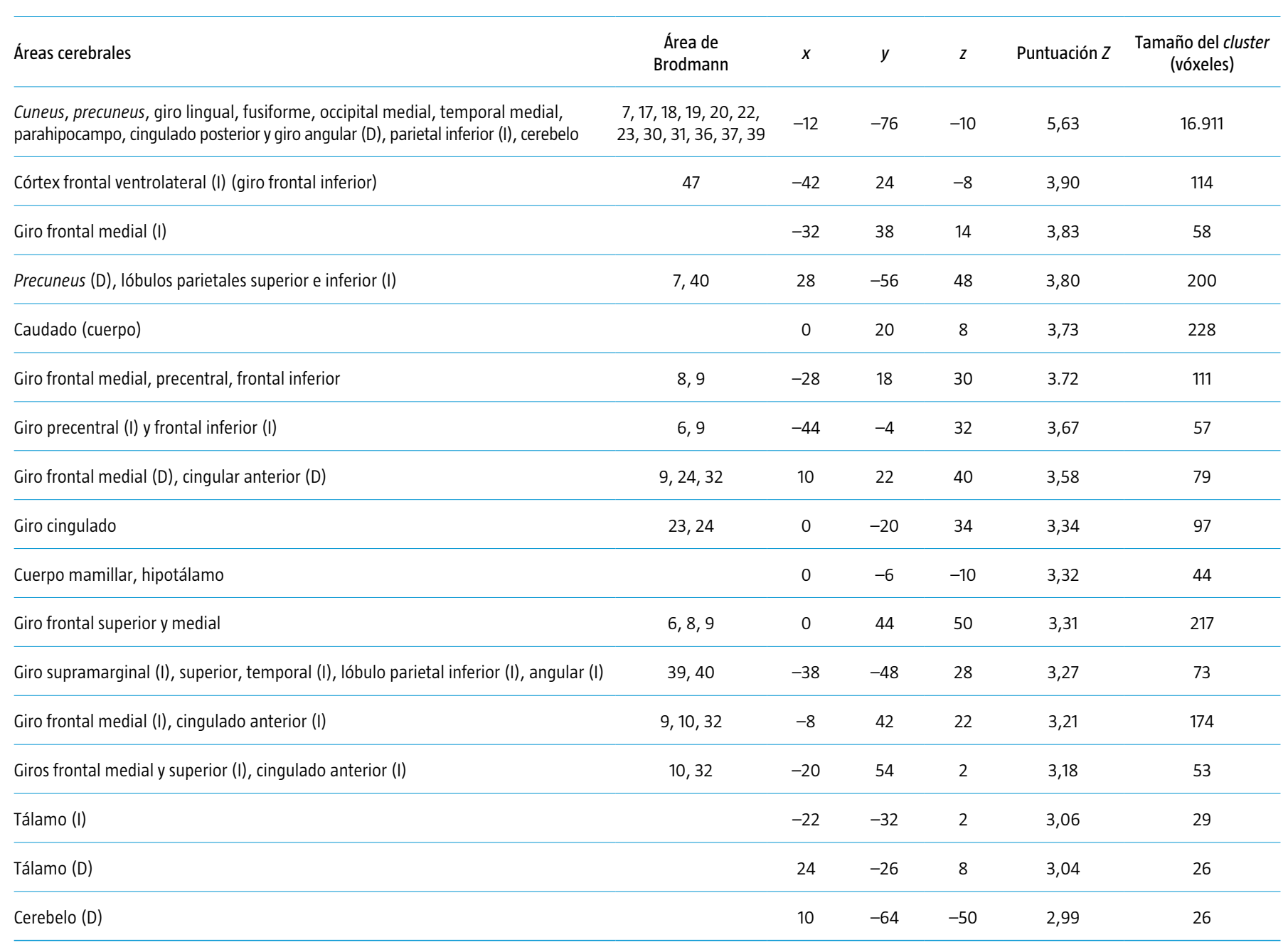

fusiforme. Por el contrario, el subgrupo de medicados tan sólo mostró mayor actividad en los giros lingual y fusiforme de forma bilateral.

\section{Relación con variables clínicas}

No se encontraron relaciones entre la activación y el nivel de depresión.

\section{Discusión}

Como muestran los resultados, ambos grupos de sujetos respondieron más lentamente cuando se les obligó a monitorizar un estímulo en la condición de control inhibitorio (go/no go), sin que se produjeran diferencias significativas entre ellos en la ejecución de la tarea. Por lo que se refiere a las áreas cerebrales activadas por el paradigma de RMf, los participantes del grupo de control mostraron activación en varias regiones del circuito frontoestriadotalámico, como el córtex prefrontal superior, inferior y medial, el núcleo caudado y amplias áreas del córtex posterior. Estos resultados coinciden parcialmente con los obtenidos en estudios previos, que han asociado el procesamiento de la magnitud de recompensa después de las respuestas con el estriado dorsal $[28,29]$, mientras que otros relacionan el control inhibitorio con la activación del córtex prefrontal inferior derecho [30,31]. La comparación de la activación durante la ejecución de la tarea en RMf entre ambos grupos mostró que los pacientes 
Tabla IV. Regiones que mostraron activación cerebral durante la ejecución de la tarea de control inhibitorio con contingencias de reforzamiento en el grupo de pacientes con trastorno obsesivo-compulsivo.

\begin{tabular}{|c|c|c|c|c|c|c|}
\hline Áreas cerebrales & $\begin{array}{l}\text { Área de } \\
\text { Brodmann }\end{array}$ & $x$ & $y$ & $z$ & Puntuación $Z$ & $\begin{array}{l}\text { Tamaño del cluster } \\
\text { (vóxeles) }\end{array}$ \\
\hline $\begin{array}{l}\text { Giro lingual, cuneus, fusiforme, precuneus, occipital medial, parahipocampo, } \\
\text { lóbulo parietal inferior y superior (I), giro temporal medial, cíngulo posterior, } \\
\text { giro angular, cerebelo }\end{array}$ & $\begin{array}{c}7,17,18,19,20 \\
23,30,31,36,37 \\
39,40\end{array}$ & -26 & -72 & -12 & 5,71 & 13.762 \\
\hline Giro frontal medial (D), cingular (D) & 9 & 6 & 38 & 32 & 3,31 & 25 \\
\hline Giro frontal medial y superior (D) & 8,9 & 36 & 32 & 40 & 3,36 & 61 \\
\hline Giro medial y precentral (D) & 9 & 34 & 8 & 42 & 3,38 & 29 \\
\hline
\end{tabular}

activaron en menor medida el cuerpo del caudado de forma bilateral, el cíngulo anterior izquierdo y los giros superior y medial frontal, mientras que tan sólo se encontraron mayores activaciones en el grupo de pacientes en el giro lingual y el fusiforme izquierdos, el lóbulo parietal inferior y el cuneus y precuneus derechos.

Estos resultados podrían alinearse con los obtenidos en estudios previos, que muestran diferencias de actividad cerebral en estas áreas al realizar tareas como la monitorización de estímulos, la inhibición de respuestas prepotentes [26] o en tareas go/no go [32], áreas que coinciden con las halladas tradicionalmente como implicadas en la patogenia del TOC. Así, el circuito corticoestriadotalámico se ha propuesto en numerosos estudios como parte de la explicación etiológica de la enfermedad obsesiva, además de su relación con las funciones ejecutivas, especialmente con la inhibición de respuesta $[13,16]$. Otros resultados en esta línea son los encontrados por Rauch et al [33], quienes, tras aplicar una tarea de aprendizaje implícito a una muestra reducida de pacientes con TOC, observaron que éstos exhibían una menor activación frontoestriatal y, además, utilizaban regiones alternativas, como el hipocampo, tal vez para compensar su déficit frontroestriatal.

Del mismo modo, usando una versión de la torre de Londres, van den Heuvel et al [34] encontraron una actividad reducida en el córtex prefrontal dorsolateral y en el núcleo caudado en el grupo de pacientes, al tiempo que éstos mostraban una mayor actividad compensatoria en las regiones prefrontal ventromedial, córtex cingulado y giro parahipocampal. Asimismo, la hipótesis del grupo de Damasio [35] también apunta que la región ventromedial de la corteza prefrontal integra los diferentes facto- res implicados en la toma de decisiones. Roth et al [17] encontraron también que los sujetos con TOC mostraban hipoactividad del circuito frontoestriadotalámico durante la inhibición de respuestas.

Nuestros resultados son compatibles con los hallazgos obtenidos por la bibliografía previa [36-38]. Más aún, en el estudio de Remijnse et al [39], el único hasta la fecha que investiga directamente el procesamiento de recompensa en pacientes con TOC, los pacientes mostraron una menor actividad en el córtex prefrontal y en el caudado derecho (zona fronteriza con el estriado ventral), al igual que los resultados obtenidos en nuestro estudio, todo lo cual nos llevaría a concluir que los pacientes con TOC muestran un patrón de activación cerebral alterado con respecto a los sujetos normales en una tarea de procesamiento de la recompensa que implica conflicto cognitivo, y que este patrón se concreta en una menor actividad de estructuras, como el córtex prefrontal superior y medial derechos, el córtex cingulado anterior izquierdo y ambos núcleos caudados, que ocurre en ausencia de diferencias significativas en la ejecución de la tarea de control inhibitorio.

Estos hallazgos acerca de la hipoactivación de las vías frontoestriales durante tareas cognitivas en el TOC deben ponerse en relación con los relativamente consistentes hallazgos de hiperactivación de estas mismas regiones durante la provocación de síntomas $[13,40]$. Se ha argumentado que la hiperactividad crónica de los circuitos frontoestriadotalámicos, que es responsable de los síntomas obsesivos y compulsivos, podría provocar que las regiones cerebrales implicadas en estos circuitos acabaran estando menos disponibles para llevar a cabo tareas cognitivas [41]. Esta afirmación está en consonancia con un estudio que mostró que el tratamiento 
farmacológico en el TOC lleva a una reducción de la activación frontoestriatal previamente incrementada durante una tarea de provocación de síntomas, mientras que aumenta la previamente reducida activación frontal observada durante la ejecución de una tarea cognitiva, como es el Stroop [42]. Nuestros resultados también muestran una menor actividad del cíngulo anterior izquierdo, el córtex frontal, el tálamo y la cabeza del caudado, al comparar los pacientes medicados frente a los no medicados durante la ejecución de la tarea de control inhibitorio, lo que también podría interpretarse en este sentido, así como en relación con estudios previos que muestran que el tratamiento con inhibidores selectivos de la recaptación de serotonina reduce la hiperactividad de los circuitos frontoestriadotalámicos en los pacientes con TOC [43].

Como parecen indicar algunos estudios [16], los procesos inhibitorios podrían ser el sustrato cognitivo de los síntomas mayores del TOC, por lo que la investigación en esta área podría resultar fundamental para aclarar la etiología de este trastorno. Menzies et al [44] estudiaron las diferencias en ejecución en pacientes y familiares de primer grado de sujetos con TOC en una tarea de inhibición de respuesta (stop-signal task) con respecto a un grupo normal, encontrando que los fallos conductuales en la tarea stop-signal ocurrían predominantemente en pacientes y familiares, y se asociaban a una reducción de la materia gris en las regiones orbitofrontal y frontal inferior derecha y a un aumento en el cíngulo, parietal y regiones estriatales. Las conclusiones a las que llegan apuntan a que la variación estructural en los sistemas cerebrales relacionados con el control inhibitorio motor podrían mediar el riesgo genético para padecer un TOC, lo que representa, según los autores del estudio, la primera evidencia de un endofenotipo neurocognitivo [45].

La mayoría de estudios sugiere que el córtex orbitofrontal, el cingulado anterior, los ganglios basales y el tálamo están implicados en la patogénesis del TOC, pero han tenido dificultades para integrar los hallazgos psicológicos y los neuroanatómicos [46]. El hecho de no encontrar diferencias significativas en la ejecución de la tarea se debe, muy probablemente, a su escasa dificultad frente a otras tareas, como la que emplean Menzies et al [44], y a la diferencia en la potencia de los análisis estadísticos. En todo caso, y precisamente por esta ausencia de diferencias conductuales, resultaría que las diferencias en activación cerebral encontradas habrían de ser la consecuencia de un distinto patrón de funcionamiento cerebral ante esta tarea. Los resultados del presente estudio aportan evidencia acerca de las
Figura 2. Activaciones durante la tarea de control inhibitorio. a) Activaciones en el grupo control; b) Diferencias en activación entre ambos grupos. Todas las activaciones con un umbral de $p<0,005$, sin corregir. Tamaño del cluster: 10 vóxeles mínimo.

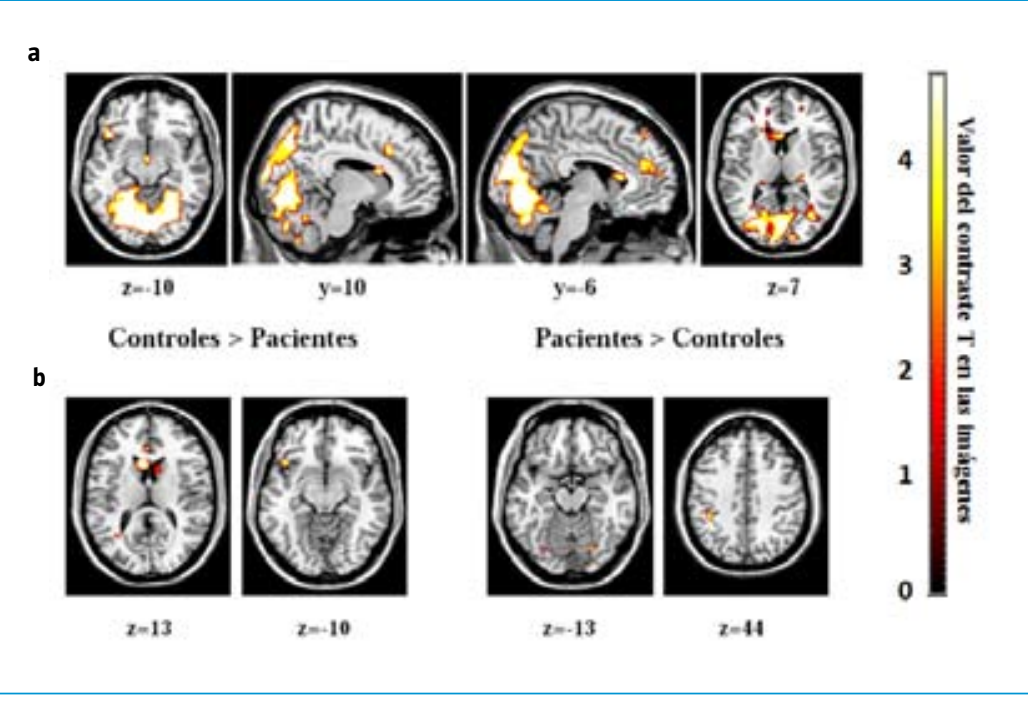

diferencias en activación cerebral de los pacientes con TOC en una tarea específica de procesamiento de la recompensa, y son compatibles con la investigación acerca de la importancia del control inhibitorio como parte del sustrato neurocognitivo de los síntomas mayores del TOC y de los modelos que intentan integrar los hallazgos de las neurociencias y la psicopatología [46]. La aparente incongruencia con otros estudios respecto a la ausencia de diferencias específicas en la activación del córtex orbitofrontal (característica del procesamiento de la recompensa) frente a la menor activación de otras áreas prefrontales (córtex prefrontal lateral medial y superior) encontrada en el presente estudio en los pacientes obsesivos con respecto a los normales podría indicar una activación característica ante esta tarea, que engloba tanto el control inhibitorio como el procesamiento de la recompensa a través de la exigencia de monitorizar, simultáneamente, las señales de inhibición (go/no go) y la anticipación de recompensa o castigo $[18,47,48]$. Estudios previos con este mismo paradigma [49] encontraron que diversas áreas en el córtex prefrontal lateral se activaron durante la tarea, incluyendo el giro frontal inferior, el giro frontal medio y el córtex motor.

La interpretación de los resultados mostrados en este estudio deben considerarse con cautela, dadas sus limitaciones. La primera limitación es el tamaño reducido de la muestra, por lo que no se han podido tener en cuenta las diferentes dimensiones sintomá- 
Tabla V. Regiones que mostraron diferencias significativas en la activación cerebral al comparar los grupos de pacientes entre sí y con el grupo control

\begin{tabular}{|c|c|c|c|c|c|c|}
\hline Área cerebral & $\begin{array}{l}\text { Área de } \\
\text { Brodmann }\end{array}$ & $x$ & $y$ & $z$ & Puntuación $Z$ & $\begin{array}{c}\text { Tamaño del } \\
\text { cluster (vóxeles) }\end{array}$ \\
\hline \multicolumn{7}{|l|}{ Controles $>\mathrm{TOC}$} \\
\hline Caudado (cuerpo) (I) & & -6 & 20 & 14 & 3,96 & 119 \\
\hline Caudado (cuerpo) (D) & & 10 & 16 & 16 & 3,59 & 40 \\
\hline Giros medial y superior (D) & 8 & 2 & 44 & 50 & 2,98 & 31 \\
\hline Cíngulo anterior & 24,32 & -2 & 36 & 14 & 2,88 & 38 \\
\hline
\end{tabular}

TOC > controles

$\begin{array}{lcccccc}\text { Precuneus (D), cuneus (D) } & 18,31 & 24 & -78 & 20 & 3,27 & 26 \\ \text { Lóbulo parietal inferior (I) } & 40 & -40 & -38 & 42 & 2,98 & 33 \\ \text { Ciros fusiforme y lingual (I) } & 19 & -30 & -76 & -18 & 2,90 & 27\end{array}$

Medicados $>$ no medicados

Giro lingual (I), fusiforme (I), occipital medial e inferior (I)

$\begin{array}{llllll}18,19 & -28 & -80 & -16 & 3,28 & 69\end{array}$

Giro lingual (D), fusiforme (R), occipital medial e inferior (D)

$18,19 \quad-24 \quad-78 \quad-16$

3,11

85

No medicados $>$ medicados

Lóbulo posterior del cerebelo (D)

Giro precentral (I)

Cingulado anterior, giro frontal medial, giro frontal superior (I)

$\begin{array}{llll}6 & -30 & -8 & 42\end{array}$

$\begin{array}{llllll}10,32 & -12 & 32 & 0 & 3,88 & 300\end{array}$

Ínsula (I)

$\begin{array}{lllll}-40 & -40 & 28 & 3,80 & 34\end{array}$

Tálamo

Precuneus, giro cingular (I)

Giro frontal inferior y temporal superior (I)

$\begin{array}{llllll}7,31 & -8 & -50 & 50 & 3,73 & 118\end{array}$

Lóbulo posterior del cerebelo (D)

Caudado (cuerpo y cabeza) (I)

Tálamo (núcleo dorsal medial) (I)

Ínsula (D)

Giro fusiforme (D)

Lóbulo anterior del cerebelo (D)

Precuneus (D)

$38,47 \quad-46 \quad 20 \quad-10$

$3,40 \quad 136$

$\begin{array}{lllll}16 & -74 & -36 & 3,38 & 49\end{array}$

Precuneus (I)

$\begin{array}{cccccc} & -10 & 12 & 12 & 3,36 & 65 \\ & -6 & -22 & 4 & 3,35 & 30 \\ 13 & 32 & 12 & 4 & 3,23 & 32 \\ 37 & -46 & -44 & -12 & 3,17 & 33 \\ & 32 & -54 & -34 & 3,16 & 25 \\ 31 & 26 & -66 & 18 & 3,12 & 40 \\ 31 & -24 & -66 & 18 & 2,97 & 26\end{array}$

TOC: trastorno obsesivo-compulsivo.

ticas que varios autores han encontrado en el TOC y que se corresponden con distintos patrones neurales parcialmente superpuestos [2,50]. El reducido tamaño de la muestra es probablemente responsa- ble directo de la baja potencia estadística del estudio. Serían necesarias más investigaciones utilizando muestras con mayor número de participantes, así como paradigmas de provocación de síntomas 
en combinación con tareas neuropsicológicas y técnicas de neuroimagen funcional. También sería deseable examinar la superposición de estos trastornos con otros del espectro obsesivo y de ansiedad, ya que algunos autores han observado que implican sustratos neurales comunes y diferentes [50]. En segundo lugar, aunque todos los pacientes cumplían criterios para el TOC como diagnóstico principal, más de la mitad presentaban comorbilidad con otros trastornos. Aun cuando ésta es una condición habitual en la clínica, conviene tomar estos resultados con precaución, ya que las diferencias encontradas pueden deberse a otras condiciones clínicas. A pesar de esta cautela, cabe señalar que en otros estudios se han utilizado muestras de pacientes con comorbilidad [32,51], y en otros no se encontraron diferencias en los patrones de activación cerebral al comparar los pacientes con diagnóstico de TOC 'puro' con aquellos otros que presentaban diagnósticos comórbidos [50]. Otra limitación a tener en cuenta es la inclusión de pacientes medicados y pacientes no medicados en nuestro estudio. Aun cuando estudios anteriores ponen de manifiesto que los hallazgos en grupos de pacientes medicados y no medicados son compatibles [52], nosotros creemos que es una cuestión que hay que señalar y tener en cuenta en la interpretación de nuestros resultados, así como en los futuros estudios en este campo.

\section{Bibliografía}

1. American Psychiatric Association. Manual diagnóstico y estadístico de los trastornos mentales, cuarta edición, texto revisado (DSM-IV-TR). Barcelona: Masson; 2000.

2. Mataix-Cols D, Rosario-Campos MC, Leckman JF. A multidimensional model of obsessive-compulsive disorder. Am J Psychiatry 2005; 162: 2193-4.

3. Breiter HC, Rauch SL. Functional MRI and the study of OCD: from symptom provocation to cognitive-behavioral probes of cortico-striatal systems and the amygdale. Neuroimage 1997; 4: 127-38.

4. Pujol J, Soriano-Mas C, Alonso P, Cardoner N, Menchón JM, Deus J, et al. Mapping structural brain alterations in obsessivecompulsive disorder. Arch Gen Psychiatry 2004; 61: 720-30.

5. Friedlander L, Desrocher M. Neuroimaging studies of obsessive-compulsive disorder in adults and children. Clin Psychol Rev 2006; 26: 32-49.

6. Luxenberg JS, Swedo SE, Flament MF, Friedland RP, Rapoport J, Rapoport SI. Neuroanatomical abnormalities in obsessivecompulsive disorder detected with quantitative X-ray computed tomography. Am J Psychiatry 1988; 145: 1089-93.

7. Robinson D, Wu H, Munne RA, Ashtari M, Alvir JM, Lerner $\mathrm{G}$, et al. Reduced caudate nucleus volume in obsessivecompulsive disorder. Arch Gen Psychiatry 1995, 52: 393-8.

8. Rosenberg DR, Averbach DH, O'Hearn KM. Oculomotor response inhibition abnormalities in paediatric obsessivecompulsive disorder. Arch Gen Psychiatry 1997; 54: 831-8.

9. Rosenberg DR, Keshavan MS. Bennet Research Award. Toward a neurodevelopmental model of obsessive-compulsive disorder. Biol Psychiatry 1998; 43: 623-40.

10. Saxena S, Brody AL, Schwartz JM, Baxter LR. Neuroimaging and frontal-subcortical circuitry in obsessive-compulsive disorder. Br J Psychiatry 1998; 173: 26-37.
11. Szeszko PR, MacMillan S, McMeniman M, Chen S, Baribault $\mathrm{K}$, Lim KO, et al. Brain structural abnormalities in psychotropic drug-naive pediatric patients with obsessive-compulsive disorder. Am J Psychiatry 2004; 161: 1049-56.

12. Gilbert AR, Mataix-Cols D, Almeida J, Lawrence N, Nutche J, Diwadkar V, et al. Brain structure and symptom dimension relationships in obsessive-compulsive disorder: a voxelbased morphometry study. J Affect Disord 2008; 109: 117-26.

13. Whiteside SP, Port JD, Abramowitz JS. A meta-analysis of functional neuroimaging in obsessive-compulsive disorder. Psychiatry Res: 2004; 132: 69-79.

14. Schlösser RG, Wagner G, Schachtzabel C, Peikert G, Koch K, Reichenbach JR, et al. Fronto-cingulate effective connectivity in obsessive compulsive disorder: a study with fMRI and dynamic causal modeling. Hum Brain Mapp 2010; 31: 1834-50.

15. Nakao T, Nakagawa A, Nakatani E, Nabeyama M, Sanematsu $\mathrm{H}$, Yoshiura $\mathrm{T}$, et al. Working memory dysfunction in obsessive-compulsive disorder: a neuropsychological and functional MRI study. Psychiatry Res 2009; 43: 784-91.

16. Chamberlain SR, Blackwell AD, Fineberg NA, Robbins TW, Sahakian BJ. The neuropsychology of obsessive compulsive disorder: the importance of failures in cognitive and behavioural inhibition as candidate endophenotypic markers. Neurosci Biobehav Rev 2005; 29: 399-419.

17. Roth RM, Saykin AJ, Flashman LA, Pixley HS, West JD, Mamourian AC. Event related functional magnetic resonance imaging of response inhibition in obsessive-compulsive disorder. Biol Psychiatry 2007; 62: 901-9.

18. Ávila C. Distinguishing BIS-mediated and BAS-mediated disinhibition mechanisms: a comparison of disinhibition models of Gray and Patterson and Newman. J Pers Soc Psychol 2001; 80: 311-24.

19. Wechsler D. Escala de inteligencia Wechsler para adultos (WAIS-III). 3 ed. Madrid: TEA; 1999.

20. First M, Spitzer R, Gibbon M, Williams J. Structured Clinical Interview for DSM-IV Axis I Personality Disorders: SCID-I. New York: American Psychiatric Press; 1997.

21. Goodman WK, Price LH, Rasmussen SA, Mazure C, Delgado P, Heninger GR, et al. The Yale-Brown Obsessive Compulsive Scale II. Validity. Arch Gen Psychiatry 1989; 46: 1012-6.

22. Sanz J, Perdigón LA, Vázquez C. Adaptación española del inventario para la depresión de Beck-II (BDI-II): propiedades psicométricas en población general. Clínica y Salud 2003; 14: 249-80.

23. Friston KJ, Holmes AP, Worsley KJ, Poline JP, Frith CD, Frackowiak RSJ. Statistical parametric maps in functional imaging: a general linear approach. Human Brain Mapping 1995; 2: 189-210.

24. Perneger TV. What's wrong with Bonferonni adjustments. BMJ 1998; 316: 1236-8.

25. Soria-Pastor S, Padilla N, Zubiaurre L, Ibarretxe N, Botet F, Costas-Moragas $\mathrm{C}$, et al. Decreased regional brain volume and cognitive impairment in preterm children at low risk. Pediatrics 2009; 124; 1161-70.

26. Nakao T, Nakagawa A, Yoshiura T, Nakatani E, Nabeyama $\mathrm{M}$, Yoshizato C, et al. A functional MRI comparison of patients with obsessive-compulsive disorder and normal controls during a Chinese character Stroop task. Psychiatry Res 2005; 139; 101-14.

27. Talairach J, Tournoux P. Co-planar stereotaxic atlas of the human brain. 3-dimensional proportional system: an approach to cerebral imaging. New York: Thieme; 1988.

28. Delgado MR, Locke, HM, Stenger VA, Fiez JA. Dorsal striatum responses to reward and punishment: effects of valence and magnitude manipulations. Cogn Affect Behav Neurosci 2003; 3: $27-38$.

29. Knutson B, Cooper JC. FMRI of reward prediction. Curr Opin Neurol 2005; 18: 411-7.

30. Rubia K, Russell T, Overmeyer S, Brammer MJ, Bullmore ET, Sharma T, et al. Mapping motor inhibition: conjunctive brain activations across different versions of go/no-go and stop tasks. Neuroimage 2001; 13: 250-61.

31. Rubia K, Smith AB, Brammer MJ, Taylor E. Right inferior 
prefrontal cortex mediates response inhibition while mesial prefrontal cortex is responsible for error detection. Neuroimage 2003; 20: 351-8.

32. Maltby N, Tolin DF, Worhunsky P, O’Keefe TM, Kiehl KA. Dysfunctional action monitoring hiperactivates frontalstriatal circuits in obsessive compulsive disorder: an eventrelated fMRI study. Neuroimage 2005; 24: 495-503.

33. Rauch SL, Wedig MM, Wright CI, Martis B, McMullin KG, Shin LM, et al. Functional magnetic resonance imaging study of regional brain activation during implicit sequence learning in obsessive-compulsive disorder. Biol Psychiatry 2007; 61: 330-6.

34. Van den Heuvel OA, Veltman DJ, Groenewegen HJ, Catz DC, Anton JLM, Van Balkom AJ, et al. Frontal-striatal disfunction during planning in obsessive-compulsive disorder. Arch Gen Psychiatry 2005; 62: 301-10.

35. Bechara A, Damasio H, Damasio AR, Lee GP. Different contributions of the human amygdala and ventromedial prefrontal cortex to decision-making. J Neurosci 1999; 19: 5473-81.

36. Pena-Garijo J, Ruipérez-Rodríguez MA, Barros-Loscertales A. Neurobiología del trastorno obsesivo-compulsivo: aportaciones desde la resonancia magnética funcional (II). Rev Neurol 2010; 50: 541-50.

37. Pena-Garijo J, Ruipérez-Rodríguez MA, Barros-Loscertales A. Neurobiología del trastorno obsesivo compulsivo: aportaciones desde la resonancia magnética funcional (I). Rev Neurol 2010; 50: 477-85.

38. Harrison BJ, Soriano-Mas C, Pujol J, Ortiz H, López-Solá M, Hernández-Ribas R, et al. Altered corticostriatal functional connectivity in obsessive-compulsive disorder. Arch Gen Psychiatry, 2009; 66: 1189-200.

39. Remijnse PL, Nielen MA, Van Balkom AJ. Reduced orbitofrontal-striatal activity on a reversal learning task in obsessive-compulsive disorder. Arch Gen Psychiatry 2006; 63: $1225-36$

40. Simon D, Kaufmann C, Müsch K, Kischkel E, Kathmann N. Fronto-striato-limbic hyperactivation in obsessive-compulsive disorder during individually tailored symptom provocation. Psychophysiology 2010; 47: 728-38.

41. Woolley J, Heyman I, Brammer M, Frampton I, McGuire PK, Rubia K. Brain activation in paediatric obsessive-compulsive disorder during tasks of inhibitory control. Br J Psychiatry 2008; 192: 25-31.

42. Nakao T, Nakagawa A, Yoshiura T, Nakatani E, Nabeyama M,
Yoshizato C, et al. Brain activation of patients with obsessivecompulsive disorder during neuropsychological and symptom provocation tasks before and after symptom improvement: a functional magnetic resonance imaging study. Biol Psychiatry 2005; 57: 901-10.

43. Stein JD. Bases empíricas del espectro obsesivo-compulsivo. In Vallejo J, Sánchez Planell L, Díez-Quevedo C, Menchón JM, eds. Temas y retos en la psicopatología actual. Madrid: Ars Médica; 2007. p. 119-30.

44. Menzies L, Achard S, Chamberlain SR, Fineberg N, Chen $\mathrm{CH}$, Del Campo N, et al. Neurocognitive endophenotypes of obsessive-compulsive disorder. Brain 2007; 130: 3223-36.

45. Chamberlain SR, Menzies L, Hampshire A, Suckling J, Fineberg NA, Del Campo N, et al. Orbitofrontal dysfunction in patients with obsessive-compulsive disorder and their unaffected relatives. Science 2008; 321: 421-2.

46. Huey ED, Zahn R, Krueger F, Moll J, Kapogiannis D, Wassermann EM, et al. A psychological and neuroanatomical model of obsessive-compulsive disorder. J Neuropsychiatry Clin Neurosci 2008; 20: 390-408.

47. Ávila C, Torrubia R. Personality, expectations and response strategies in multiple-choice question examinations in university students: a test of Gray's hypotheses. European Journal of Personality 2004; 18: 45-59.

48. Ávila C, Torrubia R. Performance and conditioning studies. In Corr PJ, ed. The reinforcement sensitivity theory of personality. Cambridge: Cambridge University Press; 2006. p. 228-60.

49. Barrós-Loscertales A. Visualización mediante resonancia magnética estructural y funcional de las diferencias individuales de sensibilidad a la recompensa. Castelló: Universitat de Castelló; 2007. URL: http://www.tdx.cat/TDX-1018107100203. [18.03.2011].

50. Mataix-Cols D, Wooderson S, Lawrence N, Brammer MJ, Speckens A, Phillips ML. Distinct neural correlates of washing, checking, and hoarding symptom dimensions in obsessive-compulsive disorder. Arch Gen Psychiatry 2004; 61: 564-76.

51. Fitzgerald KD, Welsh RC, Gehring WJ, Abelson JL, Himle JA, Liberzon I, et al. Error-related hyperactivity of the anterior cingulate cortex in obsessive compulsive disorder. Biol Psychiatry 2005; 57: 287-94.

52. Adler CM, McDonough-Ryan P, Sax KW, Holland SK, Arndt S, Strakowski SM. fMRI of neuronal activation with symptom provocation in unmedicated patients with obsessive compulsive disorder. J Psychiatr Res 2000; 34: 317-24.

\section{Involvement of the thalamic-cortical-striatal circuit in patients with obsessive-compulsive disorder during an inhibitory control task with reward and punishment contingencies}

Introduction. Recent neuroimaging studies conducted on obsessive-compulsive disorder (OCD) show alterations in the fronto-thalamic-striatal circuit, which would give rise to an executive dysfunction. This could be the neurocognitive substrate underlying the main symptoms of OCD, i.e. obsessions and compulsions.

Subjects and methods. Both brain activity and behavioural performance of a group of 13 patients with OCD were compared with a control group of 13 healthy subjects by means of functional magnetic resonance imaging while performing an inhibitory control task with reward and punishment contingencies. The effects of medication were also analysed.

Results. The intra-group analyses showed a longer reaction time during the go/no go condition in both groups, although there were no differences between the groups in the performance of the task. With regard to this task, significant activation of large areas of the cerebellum and the occipital, temporal and parietal lobes was observed in the healthy subjects. In comparison with the controls, the obsessive patients showed lower activation in the right-side frontal medial and superior gyri, the anterior cingulate cortex and the caudate nucleus, and greater activation in the inferior parietal convolution and the fusiform gyrus. The effects of medication were found in the frontal cortex and basal structures.

Conclusions. These results agree with the argument claiming that the dysfunction in the corticostriatal system in OCD is associated to diminished brain activity in response to cognitive tasks.

Key words. Cognitive task. Functional neuroimaging. Inhibitory control. Obsessive-compulsive disorder. 